\title{
Employee engagement and its relation to hospital performance in a tertiary care teaching hospital
}

\author{
Srinivas Goud Bulkapuram*1 ${ }^{* 1}$ Laxmitej Wundavalli ${ }^{2}$, Kanthi Sagar Avula ${ }^{3}$, T Reddy $\mathrm{K}^{4}$ \\ ${ }^{1}$ Kamineni Hospitals, L.B. Nagar, Hyderabad, India \\ ${ }^{2}$ All India Institute of Medical Sciences, New Delhi, India \\ ${ }^{3}$ Insurance Medical Services, Govt. of Telangana, India \\ ${ }^{4}$ Nizam's Institute of Medical Sciences, Hyderabad, India
}

Received: April 30, 2014

DOI: $10.5430 /$ jha.v4n1p48
Accepted: November 4, 2014 Online Published: January 12, 2015

URL: http://dx.doi.org/10.5430/jha.v4n1p48

\begin{abstract}
Introduction: Employees in modern organizations are expected to be proactive, show initiative, and take responsibility for their own professional development and to be committed to high quality performance standards. Human resource in healthcare is one of the key components which is scarcely available and needs to be utilized in an efficient way. Assessment of employee engagement and identifying the key areas for work place improvement are important to be able to deliver timely and quality care.

Methods: A cross-sectional survey was conducted at the Nizam's Institute of Medical Sciences, India, in the year 2012-13, that included all the permanent employees of the institute except class IV employees. The required sample size was calculated as 286. A semi structured socio-demographic proforma was designed. Employee Experience Survey used in the current study is based on Ontario Hospital Association's Quality Healthcare Workplace Model. Engagement scores were categorized into low, medium and high categories. Correlation analysis was done to identify the association between engagement score and both the outcomes: patient centered work environment (PCWE) and patient safety culture (PSC).

Results: The mean employee engagement score in the study hospital was $20.98(S D=3.15)$ which falls in the medium level. $40.6 \%$ of employees were in low engagement group, 39.9\% were in medium engagement group and $19.5 \%$ were in high engagement group. The mean PCWE score was $22.78(S D=3.19)$, and mean PSC was $21.62(S D=3.99)$ and there was a positive and significant association between employee engagement $v s$. PWCE and employee engagement $v s$. PSC.

Conclusion: The study shows a significant association between employee engagement and the key hospital outcomes, PCWE and PSC. A further analysis of the engagement survey data is required to test the direction of association and to determine the change in their relationships with respect to time. National benchmarks for such outcome measures need to be developed to enable the hospitals nationwide to assess and compare the work environments.
\end{abstract}

Key Words: Employee engagement, Hospital performance, Patient safety culture, Patient centered work environment

\section{Introduction}

Employees in modern organizations are expected to be active at work place, show initiative, and take responsibility for their own professional development and to be committed to high quality performance standards. The modern organi- zations need employees who feel energetic and dedicated i.e., who are engaged with their work. Therefore the past decade has witnessed a sharp rise in scientific studies on engagement. ${ }^{[1]}$ The focus on the subjective experience of work is common in both traditional and newer concepts but the traditional concepts fail to capture the distinct value added

*Correspondence: Srinivas Goud Bulkapuram; Email: bulka.srinivas@gmail.com; Address: Kamineni Hospitals, L.B. Nagar, Hyderabad, India. 
by the new concept of work engagement. ${ }^{[2]}$

\subsection{The concept of employee engagement}

There has been an increasing interest and research in the area of employee engagement in recent years. Employee engagement is a new and emerging area in employee relations and motivation. Many researchers believe that in ensuring employee motivation and productivity, employee engagement is one of the most fundamental concepts. Various aspects of employee engagement have been combined giving a definition of Employee Engagement as; "The extents to which employees thrive at work, are committed to their employer, and are motivated to do their best, for the benefit of themselves and their organization"(Defined by psychologists Stairs \& Galpin 2006). ${ }^{[3]}$

\subsection{Engagement in healthcare setting}

In healthcare, the concepts of employee engagement are being laid emphasis on and are evolving. The focus is being directed on developing better workplace environments. The US Joint Commission on the Accreditation of Healthcare Organizations linked high-quality care and healthy workplaces in this way: "A healthy work environment is one in which workers will be able to provide high quality care and one in which worker health and patients' care quality are mutually reinforcing". Healthcare organizations that have highly engaged employees provide quality and timely care that imparts greater value to their clients and have better financial performance according to research conducted in England's National Health Service. ${ }^{[4]}$

\subsection{Engagement and performance}

Many employers understand that business outcome depends on the human resource that promotes organizational objectives. It is estimated that disengaged employees cost U.S. organizations a significant amount of money - between $\$ 250$ and $\$ 350$ billion a year. According to a survey done around 10 years ago of about 600 CEOs from countries around the world, improving employee engagement is one of the most important problems being faced by management. ${ }^{[5]}$ There has been increasing evidence that success of any organization depends on the level of employee engagement. A recent CIPD survey in 2006 of 2,000 employees in Great Britain found that only $30 \%$ of the employees were engaged at their work. $^{[3]}$

\subsection{Patient safety culture}

The first step in transforming an organizational culture towards one that prioritizes patient safety is to understand the current state of an organization's culture. ${ }^{[6]}$ Patient safety surveys were developed by the researchers to help assessment of safety-orientation in organizational cultures. A survey aimed at staff perceptions of patient safety culture, the Hospital Survey on Patient Safety Culture, was piloted in the Veterans Health Administration and endorsed by the Agency for Healthcare Research and Quality (AHRQ). This survey targets both clinical and administrative employees' attitudes and beliefs about patient safety within and across departments, leadership commitment to safety goals, and team effectiveness. ${ }^{[7]}$ The Agency for Healthcare Research and Quality's Hospital Survey on Patient Safety has recently been expanded for use in ambulatory care organizations that are affiliated with or members of integrated health systems.

\subsection{Patient centred care}

Health care organizations are striving to improve quality by refocusing organizational policy and care delivery centered around the patient, bolstered by evidence for benefit in clinical outcomes, patient experiences and a business case that helps in heading toward "patient-centered care". ${ }^{[8,9]}$ The overall quality of care received by the patient can be improved by enhancing the patient care experience, with "patient-centeredness" included as a dimension of quality in its own right. ${ }^{[8,10]}$

The data pertaining to employee engagement particularly in healthcare setting is relatively low in developing countries due to lack of research in this direction which is important to be able to deliver quality healthcare services. Lots of research needs to take place in such countries to measure the employee engagement, identify key driving forces of engagement and implement the changes to give better results. Even in the developed countries most of the researchers have measured and identified the levels of employee engagement, but there is limited literature on the implementation aspects of improving engagement. There is a need to develop result oriented approaches to improving engagement in healthcare organizations, and this can happen through constant experimentation and quantification of results.

\subsection{Context of the study}

Health care organizations are complex and highly sophisticated systems involving human resources as a key component. Human resources in healthcare are scarcely available and need to be utilized in efficient way. The current study focuses on emphasizing the importance of employee engagement in healthcare. The study examines how the levels of engagement among the employees of a tertiary care hospital are influenced by job, work environment, management and organizational factors. The study provides a measure of engagement relevant to healthcare identifies the main drivers of engagement, examines the relation of engagement with some of the key hospital outcomes.

\subsubsection{Context and background of NIMS}

The Nizam's Institute of Medical Sciences (NIMS) is a premier teaching institute providing tertiary healthcare services to the state of Telangana and adjoining states in India. 
The institute caters to about 2.50 lakh out-patients and about 35,000 in-patients annually. It performs 9,000 major operations, 8,000 minor operations and about 3,000 emergency operations annually.

\subsubsection{Staff strength}

The institute has around 2,500 dedicated personnel, which include 166 faculty, 241 Resident doctors, 19 Medical Officers, 461 Nursing Staff, 296 Para medical staff, 164 Ministerial staff and Officers, 93 Class IV employees and Security guards. In addition, there are around 1,150 contractual employees and 680 class IV employees providing services.

\subsection{Aim of the study}

\subsubsection{Aim}

To study the relationship between employee engagement and critical hospital outcomes in a tertiary care teaching hospital.

\subsubsection{Objectives}

(1) To measure the employee engagement in various dimensions in study hospital by using Employee Experience Survey (EES).

(2) To identify the critical work environment drivers of employee engagement.

(3) To study the relationship between employee engagement levels and important hospital outcomes.

\section{Methods}

A cross-sectional survey was conducted at the Nizam's Institute of Medical Sciences. The time period of the study was for one year (2012-13).

The sampling frame included all the permanent employees of the institute.

Class IV (unskilled) permanent employees were excluded from the study due to their low literacy level. The total sampling population comprised 1,115 employees. Five strata were identified: heads of departments, clinical faculty, nursing staff, technical staff and administrative staff. The acceptable margin of error chosen was 5 percent with confidence level of 95 percent. The required sample size was calculated as 286 . Probability stratified sampling technique was used to calculate sample size for the five strata identified as depicted in Table 1.

A semi structured pro forma was designed for the study with information regarding socio- demographic data like age, gender, education, domicile, religion and number of years of experience in the institute.

EES used in the current study is based on Ontario Hospital Association's Quality Healthcare Workplace Model.
The questionnaire determines engagement drivers, individual outcomes and organizational outcomes. Work environment, job characteristics and organizational supports are the engagement drivers. Engaged and proficient employees and doctors, employee and doctor health, safety and quality of life at work are individual outcomes. Quality, patient safety, low employee turnover, enrolment, organizational stature, employee productivity and costs are the organizational outcomes in the model. The EES questions the employees in 36 features of their job, training and development opportunities, their team, their supervisor, senior management and the organization supports its employees. These factors are inputs in the OHA model (see Figure 1). These factors can be regarded as upstream influences, or "drivers" of - employee engagement. EES engagement scale was constructed based on standard social science practices. Factor loadings for the six items range between 0.77 and 0.89 and Cronbach's reliability $\alpha$ is 0.92 . The Ontario Hospital Association's Quality Healthcare Workplace Model advocates that highly engaged employees are more capable than their less-engaged counterparts in achieving organizational objectives. This Survey focuses on four outcomes: employee retention, quality of patient care or services, patient safety culture and patientcentered care. However the outcomes: retention and quality of patient care or services were omitted in the current study due to insufficient data.

Table 1: The total population size and the associated sample size for each stratum

\begin{tabular}{llll}
\hline $\begin{array}{l}\text { S. } \\
\text { no }\end{array}$ & Stratum & Population & $\begin{array}{l}\text { Sample } \\
\text { size }\end{array}$ \\
\hline 1 & Heads of departments & 25 & 6 \\
2 & Clinical faculty & 167 & 43 \\
3 & Nursing staff & 461 & 118 \\
4 & Technicians & 296 & 76 \\
5 & Administrative staff & 166 & 43 \\
& Total & 1,115 & 286 \\
\hline
\end{tabular}

The EES obtains the main aspects of a patient-centered work environment (PCWE) and patient safety culture (PSC). A PCWE scale (with a range of 6 to 30, Cronbach reliability $\alpha$ of 0.88 , and factor loadings were between 0.63 and 0.81 ), and PSC scale (with a range of 6 to 30, and a Cronbach reliability $\alpha$ of 0.78 , and factor loadings were between 0.53 and 0.76 ) were obtained from Quality Healthcare Workplace Model.

Ethical approval was obtained from the NIMS Institutional Ethics Committee prior to the start of the study.

\subsection{Data collection}

The clinical faculty of the institute was contacted in person and requested for their participation in the study. The technical and nursing staff were given the EES ques- 
tionnaire and asked to return the filled in questionnaires through the technician-in-charge and nurses-in-charge of the ward/ICU/OPD/Operation theatres. The questionnaires were distributed to the administrative staff and collected by persons unrelated to the study. The collected questionnaires were placed in a common file specific to each stratum.

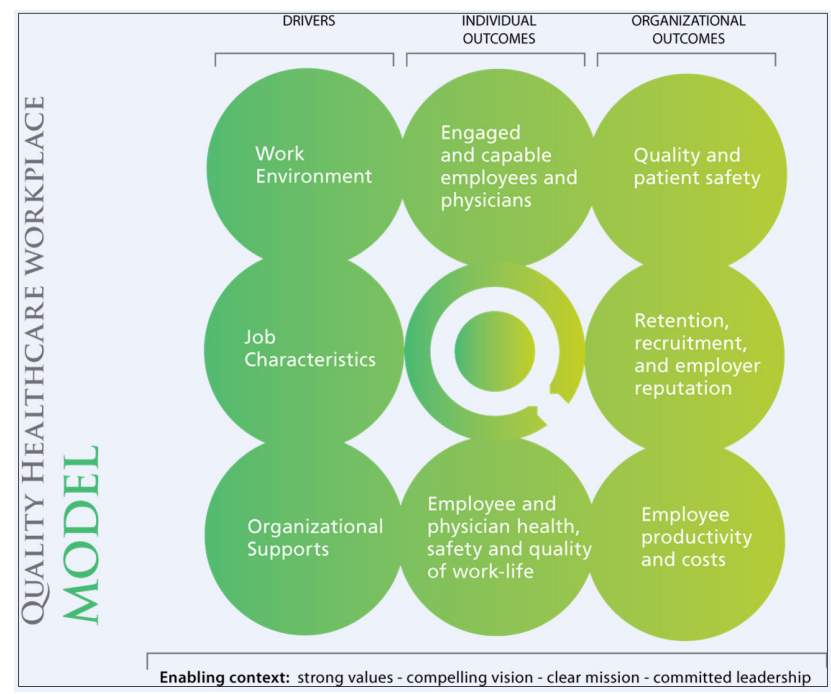

Figure 1: Quality healthcare workplace model ${ }^{[5]}$

\subsection{Analysis}

The employee engagement scores were categorized into low, medium and high levels, based on the distribution of scores. The high-engagement category comprises of individuals with responses of four or five on the five point Likert scale ( $\geq 24$ out of 36). The medium-engagement category comprises scores between 18 and 23. The low-engagement group scored 18 or lower. Logistic regression analysis was done to identify the key factors influencing the overall employee engagement scores.

Correlation analysis was done to identify if there is an association between engagement score and both the outcomes: PCWE and PSC. PCWE and PSC scores were divided into quartiles and their relationship to engagement score was determined.

Employee engagement scores were compared between the different strata of employees using Analysis of Variance.

\section{Results}

Completed questionnaires were received from 323 employees. The response rate was 69 percent.

Descriptive statistics of the study sample:

The number of participants with age $<30$ years was $13(4 \%)$, between $31-40$ years was $97(30 \%)$, $41-50$ years was $139(43 \%)$, and $51-60$ years was $74(23 \%)$.
The number of male and female participants was 104(32.20\%), and 219(67.80\%) respectively. The number of married participants was 302(93.50\%), single/unmarried participants were $17(5.26 \%)$ and divorced/separated participants were $4(1.24 \%)$. In terms of religion, the number of Hindus was 215(67\%), Muslims were 23(7\%), Christians were $80(25 \%)$ and others were $4(1 \%)$. The number of participants with 3-5 years of experience was 39(12.07\%), 6-10 years was $51(15.79 \%), 11-15$ years was $28(8.67 \%), 16-20$ years was 51(15.79\%), 21-25 years was $133(41.18 \%)$ and $>25$ years was $21(6.50 \%)$. Among the study participants administrative staff were $37(11.46 \%)$ in number, clinical faculty were $67(20.74 \%)$, nursing staff were $128(39.63 \%)$, technicians were $80(24.77 \%)$ and heads of the departments were $9(2.79 \%)$. Two participants have left this field unanswered.

The mean score (standard deviation) for job characteristics dimension was $3.50(0.91 \%)$, training and development dimension was $3.41(0.67 \%)$, work team dimension was $3.65(0.84 \%)$, Immediate supervisor dimension was 3.66(0.34\%), senior management dimension was $3.27(0.42 \%)$ and organizational support dimension was $3.49(0.60 \%)$ as shown in Figure 2.

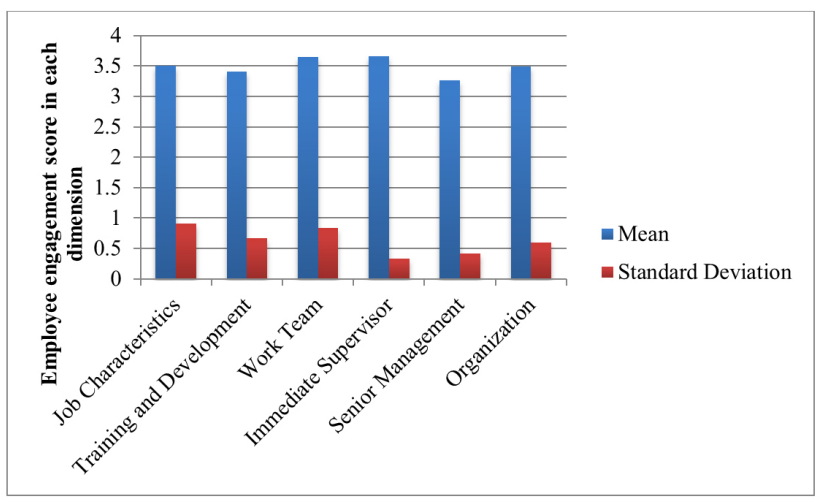

Figure 2: Means and standard deviations of overall sample with respect to each dimension of employee engagement

The mean employee engagement scores among administrative staff was $18.21(2.81 \%)$, clinical faculty was $21.29(2.86 \%)$, nursing staff was $21.19(2.70 \%)$, technicians was $21.82(3.56 \%)$ and heads of the departments was 19.49(2.18\%). The mean employee engagement score for all the employees was found to be 20.98(3.15\%) as shown in Figure 3 and Table 2. The percentage of employees in different levels of engagement in each category is shown in Table 3.

The mean scores of PCWE and PSC are depicted in Table 4 and Table 5 respectively. Spearman's correlation analysis was done to identify the relation between employee engagement and PCWE. It indicated that there is positive and significant association between employee engagement and PCWE with $r_{s}=0.817, p<.001$. Spearman's correlation 
analysis between employee engagement and PSC indicated The relationship of the levels employee engagement with that the association was also positive and was significant PCWE and PSC with respect to their quartiles is depicted in with $r_{s}=0.516, p<.001$. There was also a positive cor- Figures 4 and 5 .

relation between PCWE and PSC with $r_{s}=0.367, p<.01$.

Table 2: Means of each dimension of engagement for the five categories of staff (standard deviation in brackets)

\begin{tabular}{lllllll}
\hline S. no & Dimension & $\begin{array}{l}\text { Administrative } \\
\text { Staff }\end{array}$ & Techinicians & Nursing Staff & $\begin{array}{l}\text { Heads of the } \\
\text { Departments }\end{array}$ & Clinical Faculty \\
\hline 1 & Job Characteristics & $3.37(0.53)$ & $3.61(0.65)$ & $3.47(0.63)$ & $3.10(0.72)$ & $3.55(0.50)$ \\
2 & Training and Development & $2.41(0.68)$ & $3.42(1.01)$ & $3.54(0.63)$ & $3.38(0.82)$ & $3.72(0.57)$ \\
3 & Work Team & $3.31(0.57)$ & $3.68(0.63)$ & $3.68(0.36)$ & $3.66(0.32)$ & $3.76(0.40)$ \\
4 & Immediate Supervisor & $3.39(0.73)$ & $3.78(0.71)$ & $3.68(0.59)$ & $3.56(0.45)$ & $3.67(0.80)$ \\
5 & Senior Management & $2.5(0.85)$ & $3.71(0.84)$ & $3.38(0.83)$ & $2.78(0.26)$ & $3.08(1.04)$ \\
6 & Organizational Support & $3.24(0.79)$ & $3.73(0.82)$ & $3.44(0.62)$ & $3.02(0.74)$ & $3.51(0.54)$ \\
\hline
\end{tabular}

Table 3: Employee designation as against the levels of employee engagement

\begin{tabular}{|c|c|c|c|c|c|}
\hline \multirow{2}{*}{ Item } & & \multicolumn{3}{|c|}{ Score grade } & \multirow{2}{*}{ Total } \\
\hline & & Low & Medium & High & \\
\hline \multirow{2}{*}{ Unanswered } & Number & 2 & 0 & 0 & 2 \\
\hline & Percent within unanswered & $100.0 \%$ & 0 & 0 & 100 \\
\hline \multirow{2}{*}{ Faculty } & Number & 8 & 47 & 12 & 67 \\
\hline & Percent within Faculty & 11.9 & 70.1 & 17.9 & 100 \\
\hline \multirow{2}{*}{ Administrative staff } & Number & 33 & 4 & 0 & 37 \\
\hline & Percent within administrative staff & 89.2 & 10.8 & 0 & 100 \\
\hline \multirow{2}{*}{ Heads of the Departments } & Number & 0 & 9 & 0 & 9 \\
\hline & Percent within HOD & 0 & 100 & 0 & 100 \\
\hline \multirow{2}{*}{ Nursing staff } & Number & 13 & 64 & 51 & 128 \\
\hline & Percent in nursing staff & 10.2 & 50 & 39.8 & \\
\hline \multirow{2}{*}{ Technicians } & Number & 75 & 5 & 0 & 80 \\
\hline & Percent in Technicians & 93.8 & 6.3 & 0 & 100 \\
\hline \multirow{2}{*}{ Total } & Number & 131 & 129 & 63 & 323 \\
\hline & Percent in each score grade & 40.6 & 39.9 & 19.5 & 100 \\
\hline
\end{tabular}

Table 4: Means and standard deviation of patient centered work environment scores

\begin{tabular}{lll}
\hline Category & Mean of PCWE & $\begin{array}{l}\text { Standard } \\
\text { Deviation }\end{array}$ \\
\hline Nursing Staff & 22.09 & 3.73 \\
Clinical Faculty & 20.87 & 3.78 \\
Heads of the Departments & 22.75 & 6.16 \\
\hline
\end{tabular}

Note. Respondents associated with patients $(n=206)$.

Table 5: Means and standard deviation of patient safety culture scores

\begin{tabular}{lll}
\hline Category & Mean of PSC & $\begin{array}{l}\text { Standard } \\
\text { Deviation }\end{array}$ \\
\hline Nursing Staff & 23.16 & 3.34 \\
Clinical Faculty & 22.00 & 2.84 \\
Heads of the Departments & 24.00 & 3.16 \\
\hline
\end{tabular}

Note. Respondents associated with patients $(\mathrm{n}=206)$.

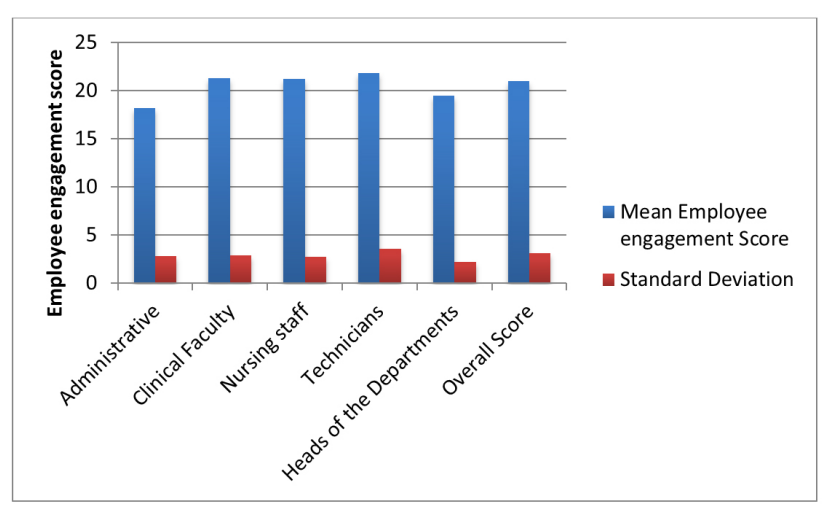

Figure 3: Means and standard deviations of each category of sample with respect to their designation

Conditional regression analysis was done to identify the key drivers of Employee engagement (see Table 6).

The following are the key engagement drivers identified in 
the study sample:

(1) I have time to carry out all my work

(2) I get recognition for good work

(3) There is opportunity to receive education/training
(4) We do not work in crisis mode

(5) Supervisor can be counted on to help with difficult tasks

(6) Supervisor provides feedback on job performance

(7) Organization provides a clean work environment

Table 6: Conditional regression analysis showing the main drivers of employee engagement

\begin{tabular}{|c|c|c|c|c|}
\hline \multirow{2}{*}{ Predictor Included } & \multicolumn{4}{|c|}{ 95\% CI for odds ratio } \\
\hline & B (SE) & Lower & Odds Ratio & Upper \\
\hline Constant & $-19.24(2.79)$ & & & \\
\hline I have time to carry out all my work & $-0.88(0.36)$ & 0.20 & 0.41 & 0.83 \\
\hline I get recognition for good work & $0.97(0.30)$ & 1.45 & 2.64 & 4.8 \\
\hline There is opportunity to receive education or training & $0.76(0.28)$ & 1.24 & 2.15 & 3.73 \\
\hline We work in crisis mode & $1.72(0.26)$ & 3.32 & 5.56 & 9.30 \\
\hline Supervisor can be approached in case of difficult tasks & $2.21(0.59)$ & 2.9 & 9.16 & 28.97 \\
\hline Supervisor provides feedback on job performance & $-1.44(0.43)$ & 0.10 & 0.24 & 0.55 \\
\hline Organization provides a clean work environment & $1.61(0.43)$ & 2.17 & 5.01 & 11.55 \\
\hline
\end{tabular}

Note. $\mathrm{R}^{2}=0.03$ (Hosmer and Lemeshow), 0.43(Cox and Snell), 0.68 (Nagelkerke); Model chi square $=180.715, p<.01$.

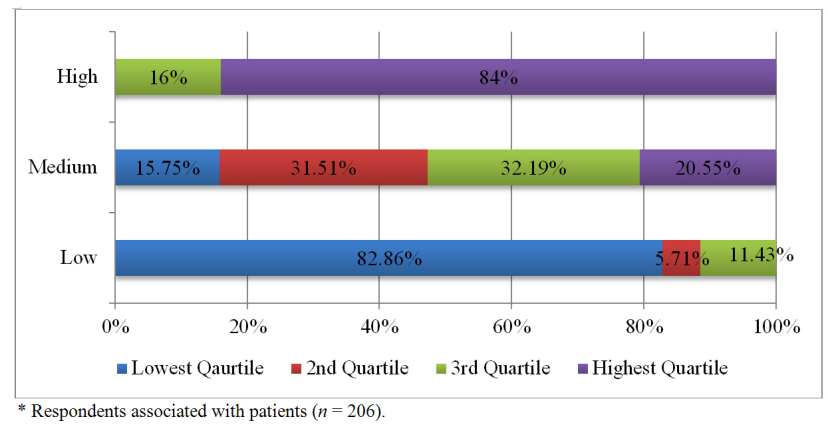

Figure 4: Patient-centred work environment scores $v s$. engagement*

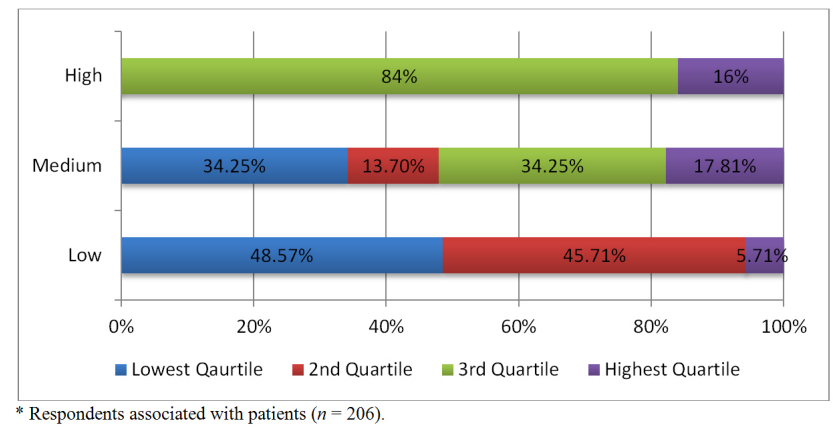

Figure 5: Patient safety culture scores $v s$. engagement*

A one way ANOVA was used to test the differences of scores in each dimension of employee engagement between each of the categories of employees. Post Hoc Tukey test was done if statistically significant differences were found in the employee engagement scores of the employees. The following were the results:

(1) Job Characteristics: There was no significant difference in the scores among the five categories of em- ployees with respect to job characteristics dimension $\mathrm{F}(4,316)=2.39, p=.051$.

(2) Training and Development: There was significant difference in the scores among the five categories of employees with respect to Training and development dimension $\mathrm{F}(4,316)=20.78, p<.01$. Tukey post hoc analysis revealed that the score in administrative staff is significantly lower compared to other staff.

(3) Work Team: There was significant difference in the scores among the five categories of employees with respect to Work Team dimension $\mathrm{F}(4,316)=5.87$, $p<.001$. Tukey post hoc analysis revealed that the scores in heads of the departments and administrative staff were significantly lower than the other three categories of staff.

(4) Immediate Supervisor: There was no significant difference in the scores among the five categories of employees with respect to job characteristics dimension $\mathrm{F}(4,316)=2.14, p=.075$.

(5) Senior Management: There was significant difference in the scores among the five categories of employees with respect to Senior Management dimension F(4, $316)=14.26, p<.001$. Tukey post hoc analysis revealed that the scores of administrative staff were significantly lower compared to technicians, nurses and clinical faculty, and the scores of technicians were significantly higher compared to other categories of staff except nurses.

(6) Organizational Support: There was significant difference in the scores among the five categories of employees with respect to Organizational Support dimension $\mathrm{F}(4,316)=4.87, p=.001$. Tukey post hoc analysis showed that the scores of technicians were significantly higher compared to the nurses, administrative staff and heads of the departments. The scores 
of clinical faculty did not differ significantly from technicians.

\section{Employee engagement score}

There is a significant difference in employee engagement score among the five categories of employees with respect to their designations with $\mathrm{F}(4,316)=11.01, p<.001$. Tukey post hoc analysis showed that the employee engagement scores in administrative staff are significantly lower than technicians, clinical faculty and nurses. The scores of the heads of the departments did not significantly differ with either of the groups.

\section{Discussion}

The purpose of the study was to assess employee engagement levels in various categories of staff in a tertiary care super specialty payment hospital run by the State Government and to establish whether any relation exists between engagement and some of the key hospital outcomes. The study followed descriptive research design employing a self administered questionnaire to the employees of NIMS.

The questions that guided the data collection and analysis were concerned with the employees' rating of work environment dimensions that led to the overall employee engagement score, PCWE and PSC. Most of the study participants found the 48 item questionnaire to be lengthy and many of them took 2-3 days to complete them. Some of the participants expressed their concerns over their anonymity with respect to the socio-demographic data sheet and didn't answer it. The nature of the questions and the utility of the results were questioned by some prospective participants and many of them refused to participate in the study. Such kind of studies assessing the work environment dimensions and engagement levels are new to the organization which could have resulted in a relatively lesser number of employees willing to participate in the study. Lack of time due to routine work was another reason mentioned by most of the participants for their inability to fill the questionnaire.

The current study showed that the maximum number of participants are lying in the low engagement group followed by medium engagement group and least in high engagement group. The engagement levels need to be improved in the study population to increase the number of employees in medium and high engagement categories.

In a study by Graham Lowe ${ }^{[5]}$ involving over 10,000 employees in 16 Ontario hospitals, the overall engagement level of the study group was in the medium engagement group with $33 \%$ of the respondents lying in the low engagement group, $39 \%$ in the medium engagement group and $29 \%$ in the high engagement group. They have considered the percentage of positive answers (rating of 4 or 5) for each of the 36 evaluative items in the questionnaire. The items with positive rankings of $\geq 60 \%$ are labelled as "strengths" and items with positive rankings of $\leq 40 \%$ are labeled as "improvement priorities" (see Table 7).

The study indicates that highly engaged work force and patient centered care tend to co-exist in the study hospital. The study also reveals that highly engaged employees tend to perceive the work environment to be more patient centric and low engagement group tends to perceive the work environment to be less patient centered. The PCWE and PSC scores are considered to be the performance indicators in the current study. The positive correlation between employee engagement and hospital performance outcomes is consistent with many other studies. Engagement or positive attitude in some form at the work place leads to improved performance outcomes. ${ }^{[11]}$ Improving the PCWE and PSC requires a team effort and the study indicates that engagement levels influence such team performance outcomes. ${ }^{[12]}$ Engaged employees often experience a positive emotional state and better health at the work place leading them to improve personal resources and contribute to organizational performance. ${ }^{[13]}$ It has also been established that highly engaged employees transfer their vigour and enthusiasm to other employees at workplace creating an overall healthy work environments and better performance outcomes. ${ }^{[14]}$ Engaged employees are also rated high by their colleagues and peers with respect to their performance. ${ }^{[15]}$ Employee ratings of engagement, organizational climate, and resources were also positively related to customer ratings of employee performance and loyalty in a study done among employees working in Spanish restaurants and hotels. ${ }^{[16]}$ High engagement levels are also related to positive performance of organizations in terms of financial returns. ${ }^{[17]}$ The current study indicates that the concept of engagement is relevant to healthcare industry and also that engagement-performance link is positive in healthcare as is the case with other industries.

\section{Conclusion}

The current survey of the hospital employees provides an opportunity to analyze the variations of employee engagement in healthcare setting. The study shows a strong association between employee engagement and the key hospital outcomes considered in the current study i.e. PCWE and PSC. However causation cannot be inferred from this association. A further analysis of the engagement survey data at a broader perspective is required to test the direction of association and to determine how their relationships change with respect to time. The reasons for high engagement could be because of being in a high performing team.

The mean employee engagement score in the study hospital was found to be 20.98 which falls in the medium engagement category. The percentage of employees in each category of engagement levels was found to be $40.6 \%$ in the low engagement group, $39.9 \%$ in the medium engagement 
group and $19.5 \%$ in the high engagement group. The study hospital needs to improve the work environment factors of engagement which in turn can lead to increase in the number of employees in high engagement group.

The overall quality improvement in the hospitals requires them to measure, report and improve the work engagement. National benchmarks for such outcome measures need to be developed to enable the hospitals nationwide to assess and compare the work environments. Hospitals must aim at achieving higher employee engagement levels as part of their continuous quality improvement measures.

Table 7: Positive ranking of work engagement dimensions

\begin{tabular}{|c|c|c|}
\hline Dimension & Questionnaire Item & Positive Responses* (\%) \\
\hline \multirow{7}{*}{ Job Characteristics } & I am able to decide how to do work & 87.96 \\
\hline & I have clear job goals/objectives & 82.10 \\
\hline & There is flexibility in Schedule/work hours & 56.79 \\
\hline & There is a balance of family/personal life with work & 51.54 \\
\hline & We have adequate resources/equipment to do work & 26.23 \\
\hline & I have time to carry out all my work & 53.09 \\
\hline & I get recognition for good work & 43.21 \\
\hline \multirow{5}{*}{ Training and Development } & There is opportunity to use skills & 57.72 \\
\hline & There is opportunity to take initiative & 52.78 \\
\hline & There is opportunity to make improvements in how my work is done & 77.16 \\
\hline & There is opportunity to receive education/training & 50.62 \\
\hline & There is opportunity to advance in career & 53.40 \\
\hline \multirow{11}{*}{ Work Team } & We work together and help each other out & 79.63 \\
\hline & People from diverse background feel welcome & 70.68 \\
\hline & We treat each other with respect & 85.19 \\
\hline & We support one another & 83.95 \\
\hline & I feel I belong to a team & 77.16 \\
\hline & I am able to make suggestions to improve work of unit/team & 75.62 \\
\hline & We collaborate well with other teams/units & 75.00 \\
\hline & Communication is open/honest & 68.21 \\
\hline & I am consulted about changes that effect unit/team & 44.44 \\
\hline & We have enough staff to handle workload & 23.46 \\
\hline & We work in crisis mode (disagree) & 29.94 \\
\hline \multirow{4}{*}{ Immediate Supervisor } & Supervisor treats you fairly & 60.80 \\
\hline & Supervisor can be counted on to help with difficult tasks & 78.40 \\
\hline & Supervisor helps access training/development & 66.67 \\
\hline & Supervisor provides feedback on job performance & 61.73 \\
\hline \multirow{4}{*}{ Senior Management } & Senior management is committed to high-quality care & 59.26 \\
\hline & Senior management is committed to improving workplace safety & 48.15 \\
\hline & Senior management communicates clearly with staff regarding goals & 48.15 \\
\hline & Senior management acts on staff feedback & 34.26 \\
\hline \multirow{5}{*}{ Organizational support } & I understand the goals of this organization & 74.38 \\
\hline & Organization provides a clean work environment & 40.43 \\
\hline & Organization promotes staff health/wellness & 40.43 \\
\hline & Organization values my work & 50.31 \\
\hline & I feel that I can trust this organization & 67.28 \\
\hline
\end{tabular}

Note. ${ }^{*}$ Combination of 4 and 5 rankings on a 5-point scale, in which, $1=$ strongly disagree, $2=$ disagree, $3=$ neither agree nor disagree, $4=$ agree, and $5=$ strongly agree.

The study revealed that $40.6 \%$ of the employees are in low engagement level which can lead to significant compromise in patient care, quality of services and lead to high staffing costs. Hospitals must adopt the reduction in engagement gap as part of their risk management. Surveys such as Em- ployee Experience Survey help the hospital managements to identify the actions required to enhance the engagement levels in low engagement groups. Persistent and focused efforts are required at the organizational level to close this engagement gap. 


\subsection{Limitations of the study}

The study includes only the subjective assessment of the hospital environment and outcome measures which could have led to incomplete assessment. The other two hospital outcome measures in the Quality Healthcare Workplace Model i.e. employee retention and work unit service quality could not be studied due to insufficient data.

\subsection{Future recommendations}

Employee engagement assessment surveys should be performed periodically in hospitals to help assess the trends and improve the work environment. Objective work environment and hospital outcome assessment tools may be used to make the assessment more accurate.

\section{References}

[1] Arnold B. Bakker, Micheal P. Leiter. Work Engagement A Handbook of Essential Theory and Research. The Psychology press; 2010. 181 .

[2] Bakker, A B, Schaufeli, W B, Leiter, M P, et al. Work engagement: An emerging concept in occupational health psychology. Work \& Stress. 2008; 22: 187-200. http://dx.doi.org/10.1080/026 78370802393649

[3] Pahris. Employee Engagement. Assessment and Evaluation. Turning Point HR solutions. October 2009.

[4] Lowe, G. How employee engagement matters for hospital performance. Healthcare Quarterly. 2012; 15(2): 29-39. PMid: 22688203. http://dx.doi.org/10.12927/hcq. 2012.22915

[5] Research Works. Partnerships for Workplace Mental Health. Employee Work Engagement: Best Practices for Employers. June 2009; 2(1).

[6] Wachter RM. The end of the beginning: patient safety five years after To Err is Human. Health Aff. 2004; W4: 534-544.

[7] Gibbons, J, Schutt R. A Global Barometer for Measuring Employee Engagement. (Research Working Group Report No. 1460-09-RR). New York: Conference Board. 2010.

[8] Ashish K, Jha M, Orav E, et al. Patients' perception of hospital care in the United States. N Engl J Med. 2008; 359: 1921-31. PMid: 18971493. http://dx.doi.org/10.1056/NEJMsa0804116

[9] Gerteis M, Edgman-Levitan S, Daley J, et al. Through the Patient's Eyes: Understanding and Promoting Patient-Centered Care. (eds). San Francisco: Jossey-Bass; 1993.

[10] Shaller D. Patient-centered care: what does it take? Report for the Picker Institute and The Commonwealth Fund. NY: The Commonwealth Fund. 2007. PMid: 17804389.
[11] Cropanzano, R, Wright, T A. When a 'happy' worker is really a 'productive' worker: A review and further refinement of the happyproductive worker thesis. Consulting Psychology Journal: Practice and Research. 2001; 53: 182-199. http://dx.doi.org/10.1037 /1061-4087.53.3.182

[12] Mullen, B, Copper, C. The relation between group cohesiveness and performance: An integration. Psychological Bulletin. 1994; 115: 210-227. http://dx.doi.org/10.1037/0033-2909.11 5.2 .210

[13] Fredrickson, BL. The role of positive emotions in positive psychology: The broaden-and-build theory of positive emotions. American Psychologist. 2001; 56: 218-226. PMid: 11315248. http: //dx.doi.org/10.1037/0003-066X.56.3.218

[14] Bakker, AB, Xanthopoulou, D. The crossover of daily work engagement: Test of an actor-partner interdependence model. Journal of Applied Psychology. 2009; 94: 1562-1571. PMid: 19916663. http://dx.doi.org/10.1037/a0017525

[15] Bakker, AB, Demerouti, E. Towards a model of work engagement. Career Development International. 2008; 13: 209-223. http: //dx.doi.org/10.1108/13620430810870476

[16] Salanova, M, Agut, S, Peiró, JM. Linking organizational resources and work engagement to employee performance and customer loyalty: The mediation of service climate. Journal of Applied Psychology. 2005; 90: 1217-1227. PMid: 16316275. http://dx.doi.org /10.1037/0021-9010.90.6.1217

[17] Despoina Xanthopoulou, Arnold B. Bakker, Evangelia Demerouti, et al. Work engagement and financial returns: A diary study on the role of job and personal resources. Journal of Occupational and Organizational Psychology. 2009; 82: 183-200. http://dx.doi.org /10.1348/096317908X285633 